\title{
MASS CONVERTED FROM THE ENERGY OF MOTION
}

This paper was downloaded from TechRxiv (https://www.techrxiv.org).

\section{LICENSE}

CC BY 4.0

SUBMISSION DATE / POSTED DATE

$27-11-2021 / 07-12-2021$

CITATION

Pandey, Vishal (2021): MASS CONVERTED FROM THE ENERGY OF MOTION. TechRxiv. Preprint. https://doi.org/10.36227/techrxiv.17087207.v1

$\mathrm{DOI}$

10.36227/techrxiv.17087207.v1 


\title{
MASS CONVERTED FROM THE ENERGY OF MOTION
}

\author{
Author Name: Vishal Pandey ${ }^{1}$ \\ ${ }^{12}$ St Thomas' College of Engineering and Technology \\ ${ }^{12}$ Kolkata, India
}

\begin{abstract}
In the General Theory of Relativity it is being introduced that the energy of motion is converted to the mass of that particle or matter or we can say that are interchangeable. It has a wide range use in the nuclear physics. The whole equation $E=\boldsymbol{m} \boldsymbol{c}^{2}$ is a relativistic mass-energy equivalence and the term "mass" is also relativistic in nature. In special relativity, however, the energy of a body at rest is determined to be $m c^{2}$. Thus, each body of rest mass $m$ possesses $m c^{2}$ of "rest energy," which potentially is available for conversion to other forms of energy. Here we initiated a equation from this if the Energy of motion has a vector form and it is in 3D space model as we know the energy of motion converted it to mass here we can do it by quantum mechanics. We think of that if the energy of motion is equal to the kinetic energy (time-independent equation from the Schrödinger equations) then we can solve the vector form of the energy and can find how much mass is being converted from the energy of motion(vector form). Here we have taken the kinetic energy from the Schrödinger equations not that from kinematics if we do then the speed of light will be equal to the velocity of that particle, which is violating the law of relativity thus I used the Schrödinger equations for simplicity. We got the equation and we have to do some calculation of the partial differentials and if the value of $M^{\prime}$ is coming to be negative then the particle doesn't exist and else we can find the mass converted and also the existence of that particle or matter of the universe. By this process, we can get the mass, the existence of matter/particle in this universe for that instance. We can use it if the energy is in the vector form and given some distance traveled in vacuum/air for some definite time we will get the desired result of mass.
\end{abstract}

Keyword: quantum physics, relativity, Schrödinger equation, mechanics.

\section{INTRODUCTION}

We know about the Einstein famous equation of the world

$E=m c^{2}$ which means that the energy and mass are interchangeable (at a very basic level), they are the different forms of the same thing. Pure energy is electromagnetic radiation — whether light or X-rays or whatever-and electromagnetic radiation travels at a constant speed of $300,000 \mathrm{~km} / \mathrm{sec}(186,000 \mathrm{miles} / \mathrm{sec})$.

When something is moving four times as fast as something else, it doesn't have four times the energy but rather 16 times the energy - in other words, that figure is squared.

If we take mass of a smallest particle as $1 \mathrm{~kg}$ then the energy would be $\mathrm{E}=90,000,000,000(\mathrm{~km} / \mathrm{sec})^{2}$ which is mindbobbling. The amount of energy of a smallest mass in the universe is very large. We can conclude that $\mathrm{E}$ is the energy of motion of any particle in this universe.

\section{THEORY}

If we think of the equation $\mathrm{E}=\mathrm{mc}^{2}$ then the only change is the energy of motion and the mass of the particle, as the velocity of light will be constant. As the two parameters $\mathrm{E}$ and $\mathrm{m}$ are both changeable then we can suppose that if the Energy of motion is being changed in the x-direction then the mass in that direction will be loose at very low value. 
Suppose we take $\mathrm{E}=$ kinetic energy of motion and $\mathrm{m}=$ mass of the particle. In this universe every particle is moving in a different manner and obvious it will lose energy. As we know the universe expands and it goes on moving in a deep space and also photon loses the energy. Assume that each galaxy is a particle we will take only one for our calculation purposes. So simply like this type we can say that as we have taken galaxy as a particle we can take a universe as a particle. No matter how we look at it he(universe/galaxy) will lose energy because it is expanding so there are lots of there(universe).

Assuming that the Energy of motion is the universe energy which it(universe) is losing in different rate of time which it is converted to the mass.

$\mathrm{E}=$ Energy loses by the universe i.e. kinetic energy of the particle(universe) in some ways.

Kinetic energy is the energy that is possesses due to motion of an object.

Kinetic energy can be time-dependent however, we will deal with the time-independent we are interested in 3D space axes criteria.

\section{CALCULATION}

As we have assumed some parameters

$\mathrm{m}=$ converted (original) mass of the particle

$\mathrm{E}=$ Kinetic energy of the particle

As we apply the value of kinetic energy from the newtonian -kinematics value then results will be:

or, $E=m c^{2}$

or, $\frac{1}{2} m v^{2}=m c^{2}$ [where, $\mathrm{m}=$ mass of the particle and $\mathrm{v}=$ velocity of the particle $]$

or, $c=\frac{1}{\sqrt{2}} v\left[\right.$ Here $\frac{1}{\sqrt{2}}$ is constant $]$

$o r, c \propto v$

if we consider this equation then every particle in this universe will have the same velocity which is equal to impossible every particle cannot have the same velocity in this universe.

or, $v=\sqrt{2} c=\sqrt{2} *\left(3 \times 10^{8}\right) \mathrm{m} / \mathrm{sec}$

$\Rightarrow 424264068.7 \cong 4.243 \times 10^{8} \mathrm{~m} / \mathrm{sec}$

Now here $\mathrm{v}>\mathrm{c}$ which is not possible. \{Violating the rules\} 
For this we will take the quantum kinetic energy of time-independent Schrödinger equation. If the energy came into the form of equation then we have to take the help of Schrödinger's equations from the conservation of energy.

The kinetic energy formula of the Schrödinger in 3D is(as we are dealing with $\mathrm{x}, \mathrm{y}, \mathrm{z}$ axes)

$$
E=m c^{2}
$$

$$
\text { Kinetic Energy }=-\frac{\hbar^{2}}{2 m} \nabla^{2}\left[\vdots \nabla^{2}=\frac{\partial^{2}}{\partial x^{2}}+\frac{\partial^{2}}{\partial y^{2}}+\frac{\partial^{2}}{\partial z^{2}}\right]
$$

Here " $\mathrm{m}$ " is the original mass before the particle' s energy of motion converted.

$$
\begin{aligned}
& \text { or },-\frac{\hbar^{2}}{2 m} \frac{\partial^{2}}{\partial x^{2}}=m_{x} c^{2} \\
& \text { or, }-\frac{\hbar^{2}}{2 m} \frac{\partial^{2}}{\partial y^{2}}=m_{y} c^{2} \\
& \text { or },-\frac{\hbar^{2}}{2 m} \frac{\partial^{2}}{\partial z^{2}}=m_{z} c^{2}
\end{aligned}
$$

Where $m_{x}, m_{y}, m_{z}$ are the masses changed in different directions by the energy of motion.

Combining all of them $(i i)+(i i i)+(i v)$ we get,

$$
\begin{aligned}
& \text { or, }-\frac{\hbar^{2}}{2 m}\left(\frac{\partial^{2}}{\partial x^{2}}+\frac{\partial^{2}}{\partial y^{2}}+\frac{\partial^{2}}{\partial z^{2}}\right)=m_{x} c^{2}+m_{y} c^{2}+m_{z} c^{2} \\
& \text { or, }-\frac{\hbar^{2}}{2 m} \nabla^{2}=\left(m_{x}+m_{y}+m_{z}\right) c^{2} \\
& \text { or, }-\frac{\hbar^{2}}{2 m} \nabla^{2}=M^{\prime} c^{2} \quad\left\{\text { where } M^{\prime}=m_{x}+m_{y}+m_{z}\right\} \\
& \text { or, }-\frac{\hbar^{2}}{2 m c^{2}} \nabla^{2}=M^{\prime}
\end{aligned}
$$


or,$-\frac{\hbar^{2}}{2 m c^{2}} \nabla^{2}=M^{\prime}$

or, $-\frac{\hbar^{2}}{2 E} \nabla^{2}=M^{\prime} \quad[$ From $(i)]$

or, $E=-\nabla^{2} \frac{\mu}{M^{\prime}}$

$\left[\therefore \mu\right.$ is a constant whose value is $\mu=\frac{h^{2}}{8 \pi^{2}}=5.56 \times 10^{-69} \mathrm{~J} . \mathrm{s}$ which can also be called as vihs constant $]$

Now the equation will be:

$M^{\prime}=-\nabla^{2}\left(|E|^{-1}\right) \hat{e} \ldots \ldots \ldots(*)$ [Here $\hat{e}$ is the unit vector along the Energy vector].

As there is a no concept of negative mass of a matter if we say it is then the matter/that particle will never exist. So it violates the Einstein's theory of general relativity. So we will transform the equation in some other manner.

From(*), squaring both sides we get,

or, $M^{\prime 2}=\nabla^{2}\left(|E|^{-2}\right)$

or, $M^{\prime}=\sqrt{\nabla^{2}\left(|E|^{-2}\right)} \quad$ [Here, required equation we get $]$

As because the energy can be in the function form and in some direction so we have taken a unit vector along it.

Energy $(\mathrm{E})$ is in function form i.e. in form of $\mathrm{x}, \mathrm{y}, \mathrm{z}$ - axis from which we can determine in which direction the particle's and how much mass is converted from the energy of motion.

For a particular time the particle will move only in one direction and in that time it will loose it's energy and also the converted-mass.

Example if a particle move $3 \hat{\imath} \mathrm{m}$ for $4 \mathrm{sec}$ and then it moves to $\hat{\jmath} m$ for $3 \mathrm{sec}$ and then again moves to $\hat{k} m$ for 1 sec for the Energy $\vec{E}=x \hat{\imath}+3 y \hat{\jmath}$ Joule and we have to find how much mass is converted from this energy.

Solution:- We will solve as the del operator we have solved and put the values of $x=3$ and $y=4$ in place of that $\mathrm{x}, \mathrm{y}$ then we get the desired result.

\section{Proper Solution:-}

Given, distance travelled by $\mathrm{x}$ is $3 \mathrm{~m}$ for $4 \mathrm{sec}$

distance travelled by $\mathrm{y}$ is $1 \mathrm{~m}$ for $3 \mathrm{sec}$

distance travelled by $\mathrm{z}$ is $1 \mathrm{~m}$ for $1 \mathrm{sec}$

as $\vec{E}=x \hat{\imath}+3 y \hat{\jmath}$ we will find $|E|=\sqrt{x^{2}+9 y^{2}}$ 
now according to formula, $M^{\prime}=\sqrt{\nabla^{2}\left(|E|^{-2}\right)}$

we will find $|E|^{2}$, so the value of $|E|^{2}$ is $x^{2}+9 y^{2}$ i.e.

$$
|E|^{2}=x^{2}+9 y^{2}
$$

Now we will go for the partial-derivative calculation.

$$
\nabla^{2}\left(|E|^{-2}\right)=\nabla^{2}\left(\frac{1}{x^{2}+9 y^{2}}\right)=\nabla^{2}\left(x^{2}+9 y^{2}\right)^{-1}
$$

Now we will find first order and then second order then we will put the value of $\mathrm{x}, \mathrm{y}, \mathrm{z}$ for their respective values and then we can find the converted mass from the energy of motion of that particle.

First order:

$$
\begin{aligned}
& \text { or, } \nabla\left(|E|^{-2}\right)=\nabla\left(x^{2}+9 y^{2}\right)^{-1} \\
& =\frac{\partial}{\partial x}\left(x^{2}+9 y^{2}\right)^{-1}+\frac{\partial}{\partial y}\left(x^{2}+9 y^{2}\right)^{-1} \\
& =\left[-1\left(x^{2}+9 y^{2}\right)^{-2}(2 x)\right]+\left[-1\left(x^{2}+9 y^{2}\right)^{-2}(18 y)\right] \\
& =-2 \frac{\{x+9 y\}}{\left(x^{2}+9 y^{2}\right)^{2}}
\end{aligned}
$$

Now we will find the second order for this equation which we had get i.e. from first order.

Second order:

$$
\begin{gathered}
\text { or, } \nabla^{2}\left(|E|^{-2}\right)=\nabla^{2}\left(-2 \frac{x+9 y}{\left(x^{2}+9 y^{2}\right)^{2}}\right) \\
=\nabla^{2}\left(-2\{x+9 y\}\left(x^{2}+9 y^{2}\right)^{-2}\right) \\
=-2\left\{\left[(x+9 y) \cdot-2\left(x^{2}+9 y^{2}\right)^{-3} \cdot 2 x+\left(x^{2}+9 y^{2}\right)^{-2}(1+0)\right]+\left[(x+9 y) \cdot-2\left(x^{2}+9 y^{2}\right)^{-3} \cdot 81 y\right.\right. \\
\left.\left.+(0+9)\left(x^{2}+9 y^{2}\right)^{-2}\right]\right\}
\end{gathered}
$$

equation.

Putting the value of $x=3, y=1$ and $z=1$ ( $z$ axis is not there) for getting the value of this differential

$$
\begin{aligned}
& =-2\left\{\left[-144\left(1.715 \times 10^{-4}\right)+\left(3.086 \times 10^{-3}\right)\right]+\left[\left(12 \times-162 \times 18^{-3}\right)+9 \times 18^{-2}\right]\right\} \\
& =-2\{(-0.02161)+(-0.3051)\}
\end{aligned}
$$


$=-2 \times(-0.32671)=(0.65442)$

So,

or, $\nabla^{2}\left(|E|^{-2}\right)=(0.65442)$

From the formula,

$$
\begin{aligned}
& \text { or, } M^{\prime}=\sqrt{\nabla^{2}\left(|E|^{-2}\right)} \\
& \text { or, } M^{\prime}=\sqrt{0.65442} \mathrm{~kg} \\
& \text { or, } M^{\prime}=0.80834 \mathrm{~kg} \text { (S.I. unit because every parameters are in S.I. units) }
\end{aligned}
$$

i.e. $0.80834 \mathrm{~kg}$ of the mass is been converted from the energy of motion of the particle moving in a particular 3D-system.

If the mass came to be negative then that particle will never exist as it violates the general theory of relativity.

\section{CONCLUSION}

In this theoretical experiment, we have concluded that the mass converted from the energy of motion of any particle moving in a 3D-space if we have the energy of motion vector and distances covered by the particle in some direction. We can also conclude that if the mass comes to the positive then the particle exist else if it comes negative then it will never exist in this universe(as it violates the general theory of relativity).

We are not taking or neglecting the value of the constant $\mu=5.56 \times 10^{-69}$ because as it been squared for removing the negative sign of the formula the value of the constant will be approximately equals to 0 . So, in this all particles shall have the same mass which is equal to 0 and it again violating some circumstances of the relativity.

So that's why the actual formula of finding the mass converted from the energy of motion (in vector field) is $M^{\prime}=$ $\sqrt{\nabla^{2}\left(|E|^{-2}\right)}$.

Here, $\vec{E}=$ Energy of motion in a 3D space vector form [Vector quantity]

$M^{\prime}=$ is the mass converted from the energy of motion[scaler quantity].

Hypothesis Statement:- If a particle is moving with a certain speed in a 3D space then it looses some energy which converted to mass and if that mass is positive then that particle will be exist in that instant time if it is negative then that particle doesn't exist.

$$
M^{\prime}=\sqrt{\nabla^{2}\left(|E|^{-2}\right)}
$$

\section{REFERENCES}

[1]. The Laplace operators, Chapter 2 from the book "Introduction to the partial differential equations" by G.B.Folland .

[2]. Charles Francis on the loss of energy in universe [https://physics.stackexchange.com/questions/588860/does-the-universe-lose-energy-whenspacetime-expands]. 
[3]. Stephan Kulla on Is time independent [https://physics.stackexchange.com/questions/485101/kinetic-energy-always-time-independent-whereis-my-mistake]. 